\title{
Estudio de caso: La gestión de la alimentación escolar en Santiago de Cali y Bogotá D.C.
}

\author{
Case study: school meals' management in Santiago de Cali and \\ Bogota
}

\author{
Mónica del Pilar Díaz ${ }^{1}$ Iván A. Montoya ${ }^{2}$ y Luz A. Montoya ${ }^{3}$
}

1 Facultad de Medicina, Universidad Nacional de Colombia. Bogotá, Colombia. mddiazb@unal.edu.co 2 Facultad de Minas, Universidad Nacional de Colombia. Medellín, Colombia. iamontoyar@unal.edu.co 3 Facultad de Ciencias Económicas, Universidad Nacional de Colombia. Bogotá, Colombia. lamontoyar@unal.edu.co

Recibido 7 Julio 2011/Enviado para Modificación 2 Octubre 2011/ Aceptado 27 Octubre 2011

\section{RESUMEN}

Objetivo La preocupación de esta investigación giró alrededor del papel del Estado frente a la problemática del hambre y la manera como gestiona en aras de combatirla, para ello se evaluó la gestión de una medida en seguridad alimentaria y nutricional en dos ciudades colombianas: la alimentación escolar en Santiago de Cali y Bogotá D.C. Métodos Se partió de un enfoque cualitativo, utilizando como técnicas de recolección de información el análisis documental, la observación participante y entrevistas a profundidad con diversos actores para ambos casos seleccionados.

Resultados No todas las medidas en la materia se encuentran bajo unos principios normativos orientados a todas las dimensiones que abarca la seguridad alimentaria y nutricional. En Santiago de Cali hay serias debilidades en la gestión que se asocian con un contexto débil en la lucha contra el hambre. Bogotá presenta una gestión encaminada a la realización del derecho a la alimentación que ha contado con un proceso institucional, donde el tema de reducir el hambre se ha vuelto un propósito.

Discusión La gestión de la medida se asocia con las características de su producto, lo cual incide en la intervención y finalmente en el estado alimentario y nutricional de la población. De esta forma, la gestión del Estado se vuelve otra dimensión de la seguridad alimentaria y nutricional.

Palabras Clave: Seguridad alimentaria, alimentación escolar, administración pública, política, nutrición de grupos vulnerables, gerencia (fuente: DeCS, BIREME).

\section{ABSTRACT}

Objective This research was aimed at ascertaining the state's role regarding hunger and how it manages to combat this matter; a food security program in two Colombian cities was thus assessed (i.e. school meals' provision in Cali and Bogota). 
Methods A qualitative approach was adopted; documentary analysis, participant observation and in-depth interviews with various actors for both selected cases were used as data collection techniques.

Results It was found that several measures taken in this area were not covered by regulatory principles aimed at covering all the dimensions of food security. Serious weaknesses in school meals' management in Cali were associated with a weak environment regarding the fight against hunger. School meals' management in Bogotá was aimed at recognizing the right to food as being supported by an institutional process where the issue of reducing hunger has become a firm purpose. Discussion School meals' program management was associated with the characteristics of its product, thereby affecting the program and the population's food and nutritional status; state management thus becomes another dimension of food security.

Key Words: Food security, school meals, public administration, politics, risk group nutrition, management (source: $\mathrm{MeSH}, \mathrm{NLM}$ ).

$\mathrm{E}$ n Colombia el 42,7 \% de los hogares padece inseguridad alimentaria y nutricional (1), lo cual refleja una condición de hambre (asumida como un estado permanente, más allá de la sintomatología aguda, la cual padece un individuo al tener una ingesta y/o aprovechamiento deficiente de nutrientes con respecto a lo requerido), lo que desdice del accionar del Estado en torno al bienestar general de la sociedad como fin del mismo (2).

Comprender la gestión para erradicar el hambre por parte del Gobierno Colombiano constituyó el propósito de esta investigación ${ }^{1}$; para ello, se planteó como objetivo evaluar la gestión de una medida en seguridad alimentaria y nutricional (SAN) en dos ciudades; entendiendo medida como una actividad dirigida a un segmento poblacional en aras de impactar alguna de las dimensiones que contribuyen a que un ciudadano ejerza su derecho a la alimentación. De esta manera, se pretendió aportar al conocimiento de las variables influyentes en la problemática del hambre, contribuir al mejoramiento de la gestión pública en ésta área y al quehacer de todos aquellos involucrados en la erradicación de este fenómeno.

\section{METODOLOGIA}

Se partió de un enfoque cualitativo, comprendiendo patrones de relación, reconociendo que el conocimiento se construye con los diferentes actores y mediado por la subjetividad de los mismos. Se inició la investigación con una

'Díaz M. [La gestión de políticas públicas en una medida para la erradicación del hambre de dos casos colombianos: La alimentación escolar en Santiago de Cali y Bogotá D.C]. Tesis de grado en Maestría en Administración; 2010. Se localiza en: Universidad Nacional de Colombia, Bogotá. 
revisión de Bibliometría Internacional, buscando modelos de estudios alrededor de la administración pública y el problema del hambre. Sin embargo, los resultados fueron escasos, de ahí que se revisaron aproximaciones a la gestión en organizaciones privadas, seleccionando la propuesta de Atamer y Calori en su libro Diagnostic et décisions stratégiques (2003), del cual se tomo el modelo para el diagnóstico de gestión, realizando la contextualización al ámbito público y al problema abordado.

De allí, se asumió la gestión como un "qué" y un "cómo" en un entorno, constituyendo estas parte fundamental de la caracterización realizada, donde el primero se refiere a la estrategia, en este caso la medida; y el segundo, a la operacionalización, la cual está asociada a los recursos, la forma en que se articulan los procesos y las fuerzas que movilizan la energía necesaria para los mismos (3).

Establecido el modelo de análisis, se seleccionaron como técnicas de recolección de información el análisis documental, la observación participante y diversas entrevistas semi-estructuradas a profundidad. Así, se realizó la triangulación de métodos y de datos obtenidos.

Se recolectaron datos en Bogotá D.C y Santiago de Cali en el primer semestre de 2010. La selección de estas, estuvo basada en el muestreo por conveniencia, sin embargo, la primera representa la población con menor grado de subnutrición, mientras que Cali se puede considerar representante del promedio de subconsumo alimentario nacional, de acuerdo a los Mapas de la situación nutricional en Colombia (4) y tomando como indicador el déficit en ingesta de proteína.

Así, para el reconocimiento del contexto, se realizó análisis documental y de forma independiente entrevistas, en Bogotá con dos miembros de la Unidad Técnica de Apoyo de la Comisión Intersectorial de Seguridad Alimentaria y Nutricional para el Distrito Capital, y en Santiago de Cali con tres integrantes de la Mesa de Seguridad Alimentaria y Nutricional del Municipio; representando dichas instancias organizaciones que lideran las acciones en el tema.

Tras el análisis, se seleccionó la medida en la que se profundizaría: la alimentación escolar, dado que en ambas ciudades se asumía como programa destacado. Una vez seleccionada, se exploró la ejecución, lo cual llevo a elegir una de las modalidades de su implementación: comida caliente tipo desayuno. Claro esto, se realizó observación participante en una sede educativa beneficiaria por cada ciudad para evidenciar cómo funcionaba. 
Se realizaron 22 entrevistas con informantes claves, contemplando representantes de las organizaciones que se involucran de forma directa en la gestión: quienes lideran la medida, la operan, la supervisan, los colegios, hasta los niños.

Ahora bien, para seleccionar los informantes se priorizaron razones de conveniencia, de manejo de información y de disponibilidad de tiempo; así como en el caso de los colegios, se contempló una sede educativa localizada en un estrato socioeconómico vulnerable que permitiera la observación del programa y que estuviera dispuesta a dar declaraciones en cada ciudad. Una vez recolectada la información, se categorizó, se identificaron patrones, se realizaron interpretaciones y se plantearon conclusiones.

\section{RESULTADOS}

El contexto

Santiago de Cali, a la fecha de recolección de información, no contaba con un plan o una política articulada y en marcha frente a SAN, siendo escasa la información que da cuenta de los antecedentes en el área, concluyendo por medio de las entrevistas que el tema es nuevo y empezó a trabajarse en el periodo de gobierno local actual, aun sin mayores resultados que mostrar.

Del lado de Bogotá, los antecedentes sobre acciones articuladas datan de 1997, concretándose en 1999 el Plan de Alimentación y Nutrición del Distrito Capital 1999-2003(5), posteriormente los respectivos planes para las 20 Localidades del Distrito, el programa "Nutrir para el Futuro" del 2001(6), "Bogotá sin Hambre" en el 2004(7), la Política Pública de Seguridad Alimentaria y Nutricional para Bogotá Distrito Capital 2007-2015 (8), el actual "Bogotá bien alimentada"(9), y todo esto con la creación de organizaciones y una institucionalidad alrededor del tema.

Así pues, el contexto del tema en Cali es débil, han pasado 18 años desde que Colombia asumió el compromiso a nivel internacional de actuar frente al hambre (Conferencia Internacional sobre Nutrición, 1992) (10) y aún no tiene una estrategia articulada y en marcha. Esto no quiere decir que no haya ninguna intervención, pero lo instaurado da cuenta de un enfoque unidimensional, centrado en actividades asumidas, principalmente, por el sector salud. Esas actividades se enraízan en un contexto donde es incipiente la organización formal frente al tema, en el que aún no hay claridad sobre el propósito de trabajar en el mismo, ni en los aspectos que los distinguen, de acuerdo a lo concluido en las entrevistas. 
Ahora, en Bogotá las acciones han sido fruto de un proceso, en el que posterior a los compromisos Internacionales, se estuvo seis años diseñando el "qué" hacer; luego se instauró esa estrategia durante cinco años, abordando en mayor medida aspectos de aprovechamiento biológico y consumo de alimentos, con una aproximación parcial a SAN, y sólo hasta hace siete años se involucran más dimensiones de SAN en la política.

La posición de Bogotá, se percibe más fuerte, hay una gestión con relación a SAN, que busca contribuir a garantizar ese derecho por parte de la sociedad y basado en eso, han establecido unos escenarios para la acción.

La medida de Alimentación escolar

En Colombia la asistencia alimentaria para escolares inicia con el Decreto 319 de 1941 bajo la dirección del Gobierno Nacional.

Para Santiago de Cali, la investigación dio cuenta que la entidad territorial asumió liderazgo frente a la medida a partir del 2002, año en el que el Gobierno Departamental transfiere recursos y fue en el 2008 cuando la estrategia se abordó desde la perspectiva de ciudad. Así pues, el programa ha estado ligado a tres formas de ejecución: la del Instituto Colombiano de Bienestar Familiar (desde el ámbito Nacional), la de la Gobernación y la del Municipio, permaneciendo las tres hasta al momento.

En Bogotá, desde el 2000 se distribuían refrigerios en los colegios oficiales con recursos del Sistema General de participaciones, del Distrito y de las Alcaldías Locales, adicional a lo que venía haciendo ICBF. Para el 2004, se incrementa la inversión del Distrito y se inicia la distribución de comida caliente, modalidades que han continuado, aunque con cambios en las características técnicas, sin que actualmente participe el ICBF. Así, en este caso hay una sola forma de organización: la liderada por el Distrito a través de la Secretaria de Educación Distrital (SED).

\section{Productos y servicios}

En Bogotá, para marzo de 2010 la alimentación escolar llegaba al 70,0 \% de estudiantes del sector oficial, cubriendo todas las sedes, pero priorizando población rural, así como cobertura de grados inferiores a superiores, excepto, para comida caliente en el que en los comedores atienden al $100 \%$ de la población escolar en los colegios donde están ubicados. 
En Bogotá el desayuno aporta entre 476 y 510 Calorías, incluyendo una bebida láctea, un alimento proteico, un cereal y una fruta diariamente. Ahora bien, en el caso observado se evidenció un producto acorde a lo planeado, con unas adecuadas prácticas de manipulación de alimentos y altos controles de calidad, entregados a primera hora del día, consumidos de forma exclusiva por los estudiantes en un espacio destinado para tal fin, con la participación de los mismos en labores de servicio social como limpieza de bandejas, alistamiento de cubiertos, entre otras, así como con el acompañamiento de docentes; aunque se reconoce que este último punto es altamente variable de una sede a otra.

Según lo indagado, prácticamente, el $100 \%$ de los niños consumen los alimentos entregados, aunque puede variar dependiendo de la aceptabilidad. Así mismo, los estudiantes refirieron satisfacción por el programa y el no sentir hambre durante la jornada como el mayor aporte de este servicio.

De otro lado, para Cali se analizó el suministro del ICBF, ya que a la fecha de recolección de datos las otras dos entidades no estaban entregando alimentos. Así pues, el ICBF a marzo de 2010 atendía el 28,0 \% de la población en sector oficial, realizando la misma priorización que se da en Bogotá.

Según los lineamientos del programa, el desayuno, el cual se denomina así por el aporte nutricional ya que también se distribuye en la tarde, debe estar conformado por una bebida con leche, Bienestarina y acompañante diario (arroz, papa, plátano, yuca, entre otros), así como huevo y fruta entera, 2 y 3 veces a la semana respectivamente, brindando entre 365 a 592 Calorías según el grupo de edad.

Sin embargo, en la observación no fue esto lo que se encontró, ya que tanto lo que se suministraba en las oportunidades que se asistió, como lo publicado en el ciclo de menús para las semanas correspondientes a las visitas, dejo ver que ni la Bienestarina, ni la fruta entera se estaban ofreciendo, el suministro de leche sólo se hacia 1 o 2 veces a la semana, y en los días que no se daba se reemplazaba por proteína de bajo valor biológico (contenida en leguminosas tales como frijol, lenteja, garbanzos, entre otros, la cual desde el punto de vista nutricional no es equivalente ), brindándose una alimentación con alto contenido de carbohidratos (ejemplo: arroz, frijoles y plátano) y bebidas a base de fruta y azúcar, cuando no se daba agua de panela.

También, se identificaron regulares practicas de manipulación de alimentos y condiciones locativas para su preparación, así como carencia de un espacio físico exclusivo para el consumo, de ahí que los estudiantes consumieran los alimentos 
de pie o sentados directamente en el suelo. En cuanto a las dinámicas, en dicha institución el consumo de alimentos se da tanto por estudiantes como por algunos docentes y otro personal administrativo, mientras que existe una baja participación de docentes en el rol de acompañamiento y nula participación de los estudiantes en la operación diaria.

Así, se evidenció y se reportó que en el horario de la mañana el consumo de alimentos es del $85,0 \%$ de los niños, mientras que en la tarde es de alrededor del $50,0 \%$. Situación que desencadena una alta cantidad de sobrante, ya que adicional a la no consumido, la institución tenía 123 raciones adicionales a su matrícula, de ahí que se suministre una porción mayor a la definida, que otro personal consume, $y$ que en frecuentes oportunidades el sobrante sea entregado a una madre de familia para su disposición.

En contraste con esto, los estudiantes afirmaron que el principal aporte del programa es que no sienten hambre durante la jornada. Sin embargo, reportaron que muchos niños no comen por vergüenza o por desconfianza de las condiciones higiénicas, lo cual fue confirmado por los docentes, quienes adicional a dichos argumentos hicieron referencia a que, en algunas oportunidades, lo ofrecido no se ajusta al habito alimentario de la población.

Gestión de la medida. Descrita las generalidades, el Cuadro 1 resume lo encontrado alrededor de las dimensiones de gestión del programa.

Cuadro 1. Síntesis de las características más relevantes en las dimensiones de gestión exploradas en el caso de la alimentación escolar en Santiago de Cali y Bogotá D.C.

\begin{tabular}{|c|c|c|}
\hline & SANTIAGO DE CALI & BOGOTÁ D.C \\
\hline $\begin{array}{l}\text { OBJETIVO DE LA } \\
\text { MEDIDA }\end{array}$ & $\begin{array}{l}\text { Tiene por objetivo el "Contribuir a } \\
\text { mejorar el desempeño académico, } \\
\text { la asistencia regular, y promover la } \\
\text { formación hábitos alimentarios } \\
\text { saludables en la población escolar" } \\
\text { (12), sin embargo, los actores } \\
\text { entrevistados no reconocen el } \\
\text { objetivo claramente. "El objetivo } \\
\text { como tal del programa está } \\
\text { enfocado en evitar la deserción } \\
\text { escolar mas que en el mejora- } \\
\text { miento nutricional" Según las } \\
\text { declaraciones del profesional de } \\
\text { ICBF entrevistado. }\end{array}$ & $\begin{array}{l}\text { Se busca contribuir al derecho a la } \\
\text { educación y a la alimentación de los } \\
\text { niños. Los diferentes actores } \\
\text { reconocen el objetivo claramente. } \\
\text { De acuerdo a lo declarado: } \\
\text { "El objetivo es retención escolar } \\
\text { pero procurando una nutrición que } \\
\text { sea adecuada para su desarrollo" }\end{array}$ \\
\hline
\end{tabular}




\begin{tabular}{|c|c|c|}
\hline & SANTIAGO DE CALI & BOGOTÁ D.C \\
\hline ADAPTACION & $\begin{array}{l}\text { Se dificulta por el carácter } \\
\text { altamente centralizado del ICBF. } \\
\text { Existen unos lineamientos desde el } \\
\text { nivel nacional que en el ámbito } \\
\text { local se modifican de forma inade- } \\
\text { cuada y se saltan lo contemplado } \\
\text { en el lineamiento. }\end{array}$ & $\begin{array}{l}\text { Se facilita porque la instancia } \\
\text { decisoria, la SED, está muy de cerca } \\
\text { desde la planeación hasta la } \\
\text { ejecución diaria. }\end{array}$ \\
\hline $\begin{array}{l}\text { FUERZAS QUE } \\
\text { MOVILIZAN LA } \\
\text { ACCIÓN }\end{array}$ & $\begin{array}{l}\text { Se considera como fortaleza evitar } \\
\text { el hambre dando alimentos, lo cual } \\
\text { se presume limita que los niños } \\
\text { lleguen a los colegios. }\end{array}$ & $\begin{array}{l}\text { Se considera como fortaleza la } \\
\text { calidad del producto, desde la ino- } \\
\text { cuidad hasta el aporte nutricional. El } \\
\text { programa se concibe por el impacto } \\
\text { que tiene no sólo en retención, sino } \\
\text { en disposición para el aprendizaje, } \\
\text { en fomento de hábitos y en mejora } \\
\text { nutricional. }\end{array}$ \\
\hline $\begin{array}{l}\text { RECURSOS } \\
\text { FINANCIEROS }\end{array}$ & $\begin{array}{l}\text { La mayoría de los actores } \\
\text { reconocen la baja inversión } \\
\text { como un obstáculo. } \\
\text { El desayuno se paga a } \$ 649 \text {, de } \\
\text { los cuales el operador debe } \\
\text { suplir materias primas, equipos, } \\
\text { menaje, pago de personal, } \\
\text { dotación y servicio de gas. Sin } \\
\text { que el Instituto asuma otros } \\
\text { costos inherentes al suministro } \\
\text { diario. }\end{array}$ & $\begin{array}{l}\text { Alta inversión financiera, Bogotá } \\
\text { paga } \$ 1488 \text { por desayuno a las } \\
\text { Cajas De Compensación Familiar } \\
\text { que operan el programa, lo que } \\
\text { involucra el pago de materias } \\
\text { primas y personal. Adicional a ello, } \\
\text { la SED adecua la infraestructura, } \\
\text { cubre el pago del servicio de gas de } \\
\text { cada comedor, los costos inherentes } \\
\text { a equipos, la dotación del personal } \\
\text { de servicio social y el menaje anual. }\end{array}$ \\
\hline $\begin{array}{l}\text { RECURSOS } \\
\text { HUMANOS }\end{array}$ & $\begin{array}{l}\text { El personal para todos los } \\
\text { procesos, excepto ejecución, no } \\
\text { es exclusivo; Existe sobrecarga } \\
\text { de trabajo desde la supervisión } \\
\text { hasta el personal operativo. Hay } \\
\text { irregularidades en las } \\
\text { condiciones laborales del } \\
\text { personal que opera el programa } \\
\text { al interior de los colegios. }\end{array}$ & $\begin{array}{l}\text { El programa cuenta con personal } \\
\text { exclusivo para los diferentes } \\
\text { procesos y en condiciones laborales } \\
\text { que no representan un obstáculo } \\
\text { para la ejecución. }\end{array}$ \\
\hline $\begin{array}{c}\text { ESTRUCTURA } \\
\text { JRGANIZACIONAL }\end{array}$ & $\begin{array}{l}\text { Se adapta al contexto de la } \\
\text { ciudad y a las características del } \\
\text { Instituto. La red de trabajo no } \\
\text { contempla, fuertemente, la } \\
\text { participación y retroalimentación } \\
\text { de la comunidad educativa. }\end{array}$ & $\begin{array}{l}\text { La red de trabajo contempla una alta } \\
\text { participación de la comunidad } \\
\text { educativa y responsables exclusivos } \\
\text { para las estrategias pedagógicas. }\end{array}$ \\
\hline PROCESOS & $\begin{array}{l}\text { Debilidad en la planeación: el } \\
\text { lineamiento expone lo ideal, } \\
\text { pero no en todos los casos se } \\
\text { hace, ni existe un responsable } \\
\text { para que se haga; desde la pla- } \\
\text { neación hay falencias en el } \\
\text { aporte nutricional en términos } \\
\text { de calidad y de hábitos } \\
\text { alimentarios. } \\
\text { Debilidad en la coordinación: } \\
\text { Hay contrariedades en las } \\
\text { estrategias desde diversos } \\
\text { sectores y poca comunicación } \\
\text { entre los actores. }\end{array}$ & $\begin{array}{l}\text { Fortaleza en la coordinación: hay } \\
\text { canales de comunicación reconoci- } \\
\text { dos y asociación de las diferentes } \\
\text { acciones emprendidas. } \\
\text { Fortaleza en la ejecución: Se } \\
\text { ofrecen servicios adicionales como } \\
\text { las estrategias pedagógicas } \\
\text { alrededor del programa y en } \\
\text { términos generales la ejecución del } \\
\text { programa es adecuada. } \\
\text { Fortaleza en el control: Los } \\
\text { mecanismos de control son varios y } \\
\text { funcionan de forma eficaz y } \\
\text { continuamente. }\end{array}$ \\
\hline
\end{tabular}




\begin{tabular}{|c|c|c|}
\hline & SANTIAGO DE CALI & BOGOTÁ D.C \\
\hline PROCESOS & $\begin{array}{l}\text { Debilidad en la ejecución: Se } \\
\text { evidenció escasas estrategias } \\
\text { adicionales y desperdicio de recursos, } \\
\text { sin que esto sea percibido por la } \\
\text { entidad líder. } \\
\text { Debilidad en el control: Hay fallas en } \\
\text { los mecanismos existentes, } \\
\text { secundarios a baja frecuencia y } \\
\text { cobertura de seguimiento; así mismo } \\
\text { el control reposa principalmente en el } \\
\text { colegio quien no conoce el } \\
\text { lineamiento del programa. } \\
\text { Debilidad en la evaluación: no hay un } \\
\text { estudio que permita dar cuenta que la } \\
\text { alimentación escolar retiene los niños } \\
\text { en el sistema educativo o que les } \\
\text { permita contrastar lo obtenido con el } \\
\text { objetivo. } \\
\text { Debilidad en el ajuste: Dado que el } \\
\text { control y la evaluación es débil y que } \\
\text { la gestión se separa de la formulación } \\
\text { en lo nacional a la implementación a } \\
\text { nivel zonal esa repuesta de ajuste es } \\
\text { más lenta. }\end{array}$ & $\begin{array}{l}\text { Debilidad en la evaluación: no hay un estudio } \\
\text { que permita dar cuenta que la alimentación } \\
\text { escolar retiene los niños. Sin embargo, han } \\
\text { adelantado estudios que les permiten medir sus } \\
\text { resultados positivos en el estado alimentario y } \\
\text { nutricional de los escolares. } \\
\text { Fortaleza en el ajuste: Al tener un control más } \\
\text { fuerte y aquella entidad que formula más cerca } \\
\text { a la implementación, hay ajustes continuos } \\
\text { basados en lo que está evidenciando. }\end{array}$ \\
\hline
\end{tabular}

\section{DISCUSIÓN}

EnSantiago de Cali hay un producto, quesi bien da saciedad, no reduce la incertidumbre con relación a falencias de nutrientes críticos. Allí, la estrategia se traduce en una ración de alimentos que mantiene aquellos factores que se vuelven contraproducentes. Es el caso de un bajo aporte de proteína y calcio, cuando hay problemas de crecimiento; un escaso suministro de frutas y verduras, existiendo un escaso hábito alimentario en torno a su consumo; un aporte alto de carbohidratos, que favorecen un suministro ante todo calórico, cuando el exceso de peso representa un problema epidemiológico; todo esto perpetuando hábitos alimentarios inadecuados que se correlacionan con el perfil de morbilidad ya reconocido en el Municipio y a nivel Nacional (13).

Allí hay una debilidad desde los supuestos, se asume que él no estar en el sistema educativo o no rendir en el mismo por hambre, está ligado a baja saciedad, buscando esta más quenutrición, lo cual en aras de impactar un problema superficial mantiene la malnutrición.

Lo anterior asociado con debilidades en la adaptación, deficiencias serias en recursos, organización deficiente y falta de armonización de esfuerzos, hace concluir que hay un proceso débil. Proceso que no se relaciona con la definición de gestión $(3,14,15,16)$, dado que no existe un propósito compartido, hay una fractura entre diseño y aplicación, una distancia de aquellos que toman decisiones que no facilita el ajuste, así como debilidades en el control y una falta de evaluación que conlleva a una escasa capacidad de maniobra hacia escenarios futuros; de ahí que se está más 
cerca de la administración, como la idea del control de planes o del actor de dirigir (17), ya que lo que se hace es dirigir un programa que otra instancia definió, y aún en ese ámbito se es débil.

Ya del lado deBogotá, su producto ataca no sólo el síntoma, sino que ha involucrado nutrientes críticos y acordes al diagnóstico de su población, abarcando desde la calidad de los alimentos hasta la promoción de adecuados hábitos alimentarios en casa, abordando más dimensiones que sólo dar comida.

Así pues, estas características se ha apalancado en un acercamiento a la gestión, con una organización en el tema, una inversión alta de recursos, procesos fuertes y articulados, ajustando con una proyección hacia el derecho a la alimentación, que redunda en un impacto positivo en el estado alimentario y nutricional y cercano al principio de eficacia en gestión. Sin embargo, ese escenario no determina una total fortaleza, hay aspectos como la evaluación y la articulación con el personal docente que aún hay que mejorar.

Ante estas dos situaciones, vale anotar la diferencia de institucionalidad alrededor de ellas. En Bogotá la fuerza política detrás del tema del hambre ha favorecido el desarrollo y posicionamiento de las acciones, existe un contexto en el que la seguridad alimentaria y nutricional se ha vuelto un escenario futuro deseado. Esto en Santiago de Cali no se ha dado, SAN todavía no hace parte de un objetivo claro a alcanzar. Debilidades y fortalezas en las reglas del juego se traducen en las características de sus medidas.

Así pues, no en todos los casos se limita la inseguridad y se garantiza los mejores servicios sociales, de acuerdo a lo que se esperaría de un Estado que busca el Bienestar (18). Hay casos, como el de Santiago de Cali, en el que a pesar del reconocimiento que en la pobreza se limita la posibilidad de elegir (19), se está perpetuando esa limitación, ofreciendo más de lo mismo. Si bien, es cierto que los dos casos se desarrollan en institucionalidades diferentes, que no los hacen comparables, si reflejan una situación de inequidad.

Con relación a la gestión, lo presentado deja ver que no en todos los casos se da. No en todas las entidades territoriales existe una visualización de la seguridad alimentaria y nutricional de la población como un escenario futuro y se realizan los ajustes en pro del mismo. De esta forma, el Estado Colombiano no ha garantizado que para toda su población se este implementando una estrategia sistemática y articulada que conduzca a la erradicación de la subnutrición y a la ejecución plena del derecho a la alimentación. 
Ahora, ya en torno a la medida, este análisis permitió observar que gestiones diferentes llevan a productos diferentes, con diferente efecto en el estado alimentario y nutricional, de ahí que la gestión en el Estado se vuelve una dimensión más que impacta en el estado alimentario y nutricional de la población •

Agradecimientos: Este artículo es el resultado de la investigación denominada "La gestión de políticas públicas en una medida para la erradicación del hambre de dos casos colombianos: La alimentación escolar en Santiago de Cali y Bogotá D.C", la cual fue desarrollada durante el año 2010 con la financiación brindada por el Fondo de Investigación de la Facultad de Ciencias Económicas de la Universidad Nacional de Colombia mediante la segunda convocatoria del año 2009.

\section{Conflicto de intereses: Ninguno}

\section{REFERENCIAS}

1. Instituto Colombiano de Bienestar familiar. Encuesta nacional de la situación nutricional en Colombia 2010. Bogotá: Instituto Colombiano de Bienestar Familiar; 2011.

2. República de Colombia. De la finalidad del Estado y de los servicios públicos. Constitución Política Colombiana. Cap. 5. Art.366. Bogotá: Imprenta Nacional; 1991.

3. Atamer T, Calori R. Diagnóstic et décisiones strategiques. Paris:Dunod; 2003.

4. Instituto Colombiano de Bienestar familiar. Mapas de la situación nutricional en Colombia. Bogotá: ICBF; 2005.

5. Bogotá, Alcaldía Mayor. Plan de Alimentación y Nutrición del Distrito Capital 1999-2003. Bogotá: Alcaldía Mayor de Bogotá; 1999.

6. Bogotá, Alcaldía Mayor. Programa Nutrir para el Futuro. Bogotá: Alcaldía Mayor de Bogotá; 2001.

7. Bogotá, Alcaldía Mayor. Programa Bogotá sin Hambre. Bogotá: Alcaldía Mayor de Bogotá; 2004.

8. Bogotá, Alcaldía Mayor. Política pública de seguridad alimentaria y nutricional para Bogotá, Distrito Capital 2007-2015. Bogotá: Alcaldía Mayor; 2007.

9. Bogotá, Alcaldía Mayor. Programa Bogotá bien alimentada. Bogotá: Alcaldía Mayor de Bogotá; 2008.

10. Organización de Naciones Unidas para la Agricultura y la Alimentación. Conferencia Internacional sobre Nutrición; 1992. [Internet]. Disponible en: http://www.fao.org/docrep/V7700T/ v7700t04.htm Consultado septiembre de 2009.

11. República de Colombia. Decreto 319 del 15 de febrero de 1941, por el cual se dictan normas sobre aporte de la Nación a los restaurantes escolares en el país. Diario Oficial No. 24.596 de 24 de febrero de 1941.

12. Instituto Colombiano de Bienestar familiar. Lineamientos técnico-administrativos y estándares para la asistencia alimentaria al escolar-programa de alimentación escolar. Bogotá: ICBF; 2007.

13. Profamilia. Encuesta Nacional de Demografía y salud 2005. Bogotá: Profamilia; 2005.

14. Bozeman B. Los fundamentos de la gestión pública estratégica. En: Bruge Q, Subirats J. (Eds). Lecturas de gestión pública. Madrid: Ministerio de administraciones públicas; 1996. p. 337402.

15. Etkin J. Gestión de la complejidad en las organizaciones. México: Oxford University Press; 2003.

16. Hughes O. La nueva gestión pública. En: Bruge Q, Subirats J.(Eds). Lecturas de gestión pública. Madrid: Ministerio de administraciones públicas; 1996. p. 103-132.

17. Romero R. El desarrollo del conocimiento y el manejo de las organizaciones. Revista INNOVAR 1998; 11: 9-16.

18. Gómez E. El Estado de Bienestar. Presupuestos éticos y políticos. Tesis Doctoral Universidad de Barcelona; 2001. [Internet]. Disponible en: http://www.tdx.cesca.es/TESIS_UB/AVAILABLE/ TDX-0425103-124025//TESISGPEDRO.pdf Consultado enero de 2010.

19. Sen A. Desarrollo y libertad. Colombia: Editorial Planeta; 2001. 